\title{
Targeting DXP synthase in human pathogens: enzyme inhibition and antimicrobial activity of butylacetylphosphonate
}

\author{
Jessica M Smith ${ }^{1}$, Nicole V Warrington ${ }^{2}$, Ryan J Vierling ${ }^{1}$, Misty L Kuhn ${ }^{3}$, Wayne F Anderson ${ }^{3}$, \\ Andrew T Koppisch ${ }^{2}$ and Caren L Freel Meyers ${ }^{1}$
}

The unique methylerythritol phosphate pathway for isoprenoid biosynthesis is essential in most bacterial pathogens. The first enzyme in this pathway, 1-deoxy-D-xylulose 5-phosphate (DXP) synthase, catalyzes a distinct thiamin diphosphate (ThDP)dependent reaction to form DXP from D-glyceraldehyde 3-phosphate (D-GAP) and pyruvate and represents a potential antiinfective drug target. We have previously demonstrated that the unnatural bisubstrate analog, butylacetylphosphonate (BAP), exhibits selective inhibition of Escherichia coli DXP synthase over mammalian ThDP-dependent enzymes. Here, we report the selective inhibition by BAP against recombinant DXP synthase homologs from Mycobacterium tuberculosis, Yersinia pestis and Salmonella enterica. We also demonstrate antimicrobial activity of BAP against both Gram-negative and Gram-positive strains (including E. coli, S. enterica and Bacillus anthracis), and several clinically isolated pathogens. Our results suggest a mechanism of action involving inhibition of DXP synthase and show that BAP acts synergistically with established antimicrobial agents, highlighting a potential strategy to combat emerging resistance in bacterial pathogens.

The Journal of Antibiotics (2014) 67, 77-83; doi:10.1038/ja.2013.105; published online 30 October 2013

Keywords: DXP synthase; isoprenoid biosynthesis; selective inhibitor; thiamin diphosphate

\section{INTRODUCTION}

The ever-present need for discovery of new antibiotics demands concerted efforts to identify novel molecular scaffolds and develop underexplored targets, and ostensibly one will perpetuate the other. A relatively new set of potential antibacterial targets comprises the methylerythritol phosphate (MEP) pathway (Figure 1) to the essential isoprenoid precursors, isopentenyl diphosphate (IDP) and dimethylallyl diphosphate (DMADP) ${ }^{1-3}$ Orthogonal to the mevalonate pathway to IDP and DMADP in humans, the MEP pathway is widespread in bacterial pathogens and offers opportunities for the development of non-toxic, broad-spectrum antibacterial agents.

An underexplored target within this pathway is 1-deoxy-D-xylulose 5-phosphate (DXP) synthase, which catalyzes the first step to form DXP from D-glyceraldehyde 3-phosphate (D-GAP) and pyruvate in a thiamine diphosphate (ThDP)-dependent manner. ${ }^{4-9}$ In many bacterial pathogens, this step is also a branch point in metabolism; DXP is a precursor to pyridoxal phosphate biosynthesis and, interestingly, also a precursor to its own essential cofactor, ThDP (Figure 1). Despite its importance in bacterial metabolism, DXP synthase is underdeveloped as a drug target. Ostensibly, this is largely due to perceived challenges in achieving selective inhibition of DXP synthase over mammalian ThDP-dependent enzymes, such as the E1 subunit of pyruvate dehydrogenase and transketolase, and few reports describe inhibitors of this enzyme. ${ }^{10-14}$ However, DXP synthase appears unique among ThDP-dependent enzymes. The Deinococcus radiodurans DXP synthase crystal structure shows a distinctive domain arrangement, ${ }^{15}$ and the active site is large compared with other ThDP-dependent enzymes. ${ }^{16}$ The unique requirement for ternary complex formation in DXP synthase catalysis ${ }^{11,17,18}$ also sets it apart from other ThDP-dependent enzymes, suggesting that selective inhibition should be possible. Indeed, our previous work $^{16,19}$ has demonstrated that selective inhibition of Escherichia coli DXP synthase is achievable with unnatural bisubstrate analogs including butylacetylphosphonate (BAP) and benzylacetylphosphonate, both bearing an acetylphosphonate mimic of pyruvate ${ }^{10,20-22}$ and a sterically demanding unnatural acceptor substrate. Presumably, sterically demanding acetylphosphonates bind in the large DXP synthase active site and react with bound ThDP, in a manner similar to methylacetylphosphonate, ${ }^{23-27}$ to form a stable phosphonolactyl-ThDP intermediate, effectively trapping the enzyme at a predecarboxylation complex (Figure 2).

${ }^{1}$ Department of Pharmacology and Molecular Sciences, School of Medicine, Johns Hopkins University, Baltimore, MD, USA; ${ }^{2}$ Deptartment of Chemistry and Biochemistry, Northern Arizona University, Flagstaff, AZ, USA and ${ }^{3}$ Center for Structural Genomics of Infectious Diseases, Northwestern Feinberg School of Medicine, Department of Molecular Pharmacology and Biological Chemistry, Chicago, IL, USA

Correspondence: Dr CL Freel Meyers, Department of Pharmacology and Molecular Sciences, School of Medicine, Johns Hopkins University, 725N Wolfe Street, WSBSB 307A, Baltimore, MD 21205, USA.

E-mail: cmeyers@jhmi.edu

Received 29 August 2013; revised 20 September 2013; accepted 30 September 2013; published online 30 October 2013 


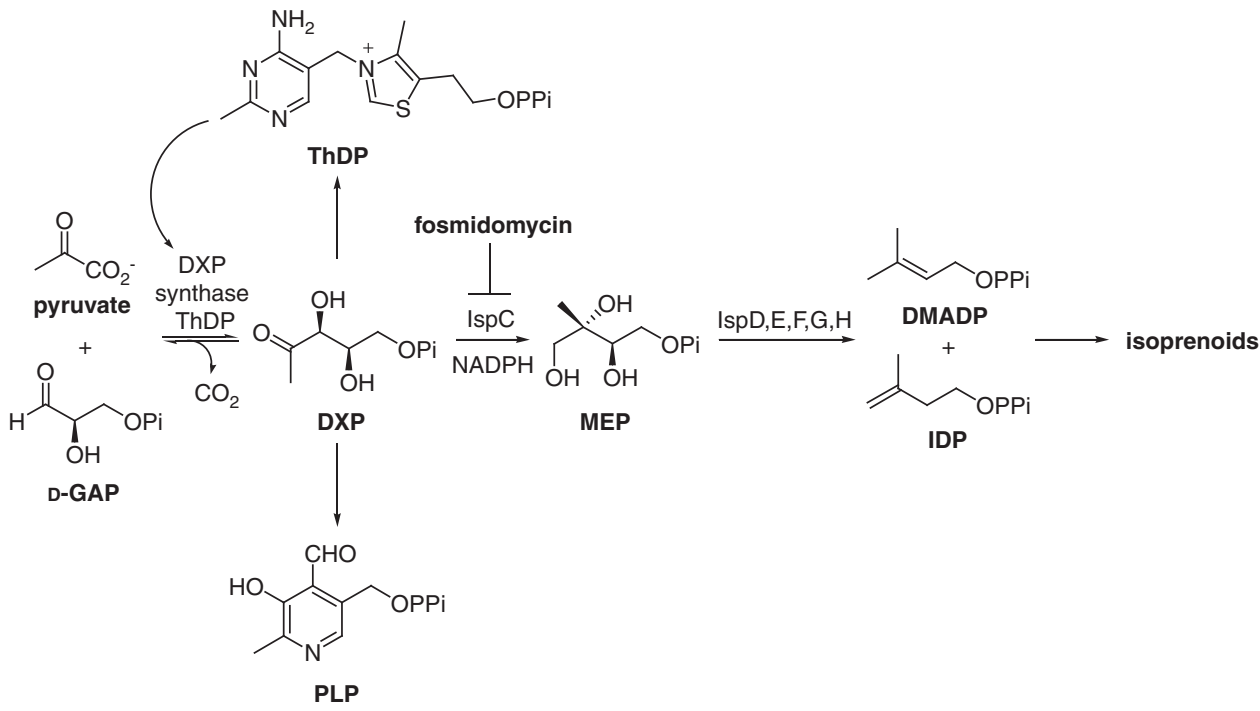

Figure 1 DXP synthase catalyzes the first step in IDP/DMADP biosynthesis and represents a branch point in bacterial metabolism.

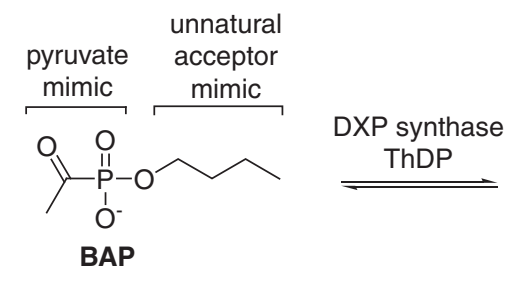

Figure 2 Proposed mechanism of selective inhibition of DXP synthase by butylacetylphosphonate.

Here, we characterize the inhibitory activity of BAP, ${ }^{19}$ against DXP synthase from Mycobacterium tuberculosis, Yersinia pestis and Salmonella enterica serovar Typhi to establish comparable inhibitory activity against this enzyme across these pathogens. Further, we have evaluated the antimicrobial activity of BAP against several bacterial pathogens. Our results indicate that BAP possesses modest antibacterial activity via a mechanism that appears to involve inhibition of DXP synthase, and drug synergism is evident with a BAP-fosmidomycin combination in E. coli. These results suggest that selective inhibitors of DXP synthase could be effective in antibiotic combinations.

\section{MATERIALS AND METHODS}

\section{General methods}

Unless otherwise noted, all reagents were obtained from commercial sources. Spectrophotometric analyses were performed on a Beckman DU800 UV/Visible spectrophotometer (Brea, CA, USA). Primers were purchased from Integrated DNA Technologies (Coralville, IA, USA). E. coli wild-type DXP synthase and the variant E370A DXP synthase were purified as described previously. ${ }^{28}$ E. coli MEP synthase (IspC) was also purified as reported previously. ${ }^{11}$ All microbial manipulation of pathogenic bacteria was conducted in a certified biosafety level 2 laboratory with all associated safety protocols. S. enterica serovar Typhimurium LT2, B. anthracis Sterne and all clinically isolated strains are maintained from an in-house bacterial strain library (NAU).
Cloning, overproduction and purification of M. tuberculosis DXP synthase (Dxs1)

Rv2682c was polymerase chain reaction amplified from H37Rv genomic DNA and ligated into the pET28a + vector (Novagen, Darmstadt, Germany), as reported previously. ${ }^{8}$ Dxs1-pET28a + was then transformed into ArcticExpress-competent cells (Agilent Technologies, Santa Clara, CA, USA). Cells harboring the overexpression plasmid were grown in LB broth at $30^{\circ} \mathrm{C}$ with shaking at 220 r.p.m. until $\mathrm{OD}_{600}=0.8$, and then cooled for $30 \mathrm{~min}$ on ice. After cooling, cultures were induced with $0.1 \mathrm{~mm}$ isopropylthiogalactoside, and growth was continued with shaking at $13{ }^{\circ} \mathrm{C}$ for $24 \mathrm{~h}$. Cells were harvested by centrifugation at 8000 r.p.m. for $10 \mathrm{~min}$, and the cell pellet was stored at $-20{ }^{\circ} \mathrm{C}$. The frozen cell pellet was suspended in lysis buffer ( $3 \mathrm{ml}$ per $\mathrm{g}$ of cells) containing $50 \mathrm{~mm}$ Tris- $\mathrm{HCl}(\mathrm{pH}=8), 5 \mathrm{~mm} \mathrm{MgCl}_{2}, 10 \%$ glycerol (v/v), $5 \mathrm{~mm}$ $\beta$-mercaptoethanol, $1 \mathrm{~mm}$ phenylmethylsulfonyl fluoride, $1 \mathrm{~mm}$ ThDP, $1 \times$ protease inhibitor cocktail, $100 \mathrm{~mm} \mathrm{NaCl}$ and $0.01 \%$ Igepal-CA630. Cells were lysed by sonication and centrifuged for $45 \mathrm{~min}$ at 18000 r.p.m. to pellet cell debris. The supernatant was incubated with nickel-nitrilotriacetic (NTA) acid resin in $20 \mathrm{~mm}$ imidazole at $4{ }^{\circ} \mathrm{C}$ for $2 \mathrm{~h}$, and Dxs1 was eluted from the resin over a stepwise gradient of 20-200 mm imidazole. Fractions containing Dxs1 (as determined by $10 \%$ SDS-PAGE) were combined and subjected to dialysis overnight at $4{ }^{\circ} \mathrm{C}$ against 1 liter of $50 \mathrm{~mm}$ Tris ( $\mathrm{pH} 8.0$ ), $10 \mathrm{~mm} \mathrm{MgCl}_{2}, 10 \%$ glycerol, $1 \mathrm{~mm}$ ThDP and $100 \mathrm{~mm} \mathrm{NaCl}$. A second dialysis was carried out against 1 liter of $50 \mathrm{~mm}$ Tris ( $\mathrm{pH}$ 8.0), $10 \mathrm{~mm} \mathrm{MgCl}_{2}, 10 \%$ glycerol, $1 \mathrm{~mm}$ ThDP, and $100 \mathrm{~mm} \mathrm{NaCl}$ and $1 \mathrm{~mm}$ for an additional $4 \mathrm{~h}$. After dialysis, protein concentration was determined using the Bio-Rad Protein Assay with bovine serum albumin as a standard (yield $1.0 \mathrm{mgl}^{-1}$ of culture). Protein was flash frozen in liquid nitrogen and stored at $-80^{\circ} \mathrm{C}$.

\section{Cloning, overproduction and purification of $Y$. pestis, and} S. enterica serovar Typhi DXP synthase

The Y. pestis and S. enterica serovar Typhi DXP synthase genes were cloned into the pMCSG28 vector using ligation-independent cloning methods as described previously, 29,30 and the resulting plasmids were transformed into Single Step KRX-competent cells (Promega, Madison, WI, USA). Cells harboring the overexpression plasmid were grown in TB broth containing $100 \mu \mathrm{g} \mathrm{ml}^{-1}$ ampicillin, $34 \mu \mathrm{g} \mathrm{ml}^{-1}$ chloramphenicol and $0.1 \% \mathrm{~L}-(+)-$ arabinose. After growth reached $\mathrm{OD}_{600}=0.6$ at $37^{\circ} \mathrm{C}$, the cultures were cooled to room temperature and induced with $0.5 \mathrm{~mm}$ isopropylthiogalactoside and $0.25 \%$ rhamnose, and shaking was continued for $16 \mathrm{~h}$. Cells were harvested, lysed and purified using the same method as described for M. tuberculosis Dxs1. 


\section{DXP synthase kinetic analysis}

Michaelis-Menten kinetic analyses were carried out as described previously ${ }^{11,19}$ with both the natural substrates (pyruvate and D-GAP) and inhibitor, BAP. BAP was synthesized as reported previously. ${ }^{19}$

\section{Antimicrobial susceptibility studies}

Using aseptic techniques, three to five isolated colonies were picked from a plate containing ATCC MG1655 E. coli and Bacillus subtilis 168 were inoculated into $5 \mathrm{ml}$ of cation-adjusted Mueller Hinton Broth (CAMHB; Sigma, St Louis, MO, USA) at $37^{\circ} \mathrm{C}$. Incubation was continued until turbidity matched McFarland turbidity standard $0.5\left(\sim \mathrm{OD}_{600}=0.10\right)^{31,32}$. Colony counts were checked after $16 \mathrm{~h}$ at $37^{\circ} \mathrm{C}$ for consistency between experiments. The standardized inoculums (McFarland $=0.5)$ contained approximately $1-2 \times 10^{8} \mathrm{CFU} \mathrm{ml}^{-1}$. The final concentration in a well (or culture tube) was $5 \times 10^{5} \mathrm{CFU} \mathrm{ml}^{-1} .31,32$ For these studies, the standardized inoculum $($ McFarland $=0.5)$ was diluted 1:100 in CAMHB and $0.25 \mathrm{ml}$ of this adjusted inoculum was added to each tube containing $0.25 \mathrm{ml}$ antimicrobial agent(s) for a final volume of $0.5 \mathrm{ml}$. These tubes were then incubated at $37^{\circ} \mathrm{C}$ for $16-18 \mathrm{~h}$, with shaking. Fractional growth was determined relative to the nodrug control, and averaged values were used. The MIC was determined to be the concentration at which growth was $\leqslant 15 \%$ of the no-drug growth controls (determined by $\mathrm{OD}_{600}$ ). The concentration of BAP evaluated for these studies ranged from 0 to $5 \mathrm{~mm}\left(0-1000 \mu \mathrm{g} \mathrm{ml}^{-1}\right)$. ATCC 70084 Mycobacterium smegmatis was evaluated in a manner similar to that outlined above, but using enriched Middlebrook $7 \mathrm{H} 9$ broth. A $24 \mathrm{~h}$ culture $\left(\sim \mathrm{OD}_{600}=0.4\right)$ was diluted to $\mathrm{OD}_{600}=0.1$ and subcultured $1: 100$ for $1 \times 10^{6}$ colony-forming units. The concentration of BAP was varied 0-10 mM $\left(0-2000 \mu \mathrm{g} \mathrm{ml}^{-1}\right)$. The $E$. coli glycerol 3-phosphate transporter knockout strain (JW2234-2) was evaluated along with the parent strain (BW25113) using the above method (Yale, Coli Genetic Stock Center, CGSC, New Haven, CT, USA). Susceptibility studies were performed for S. enterica serovar Typhimurium LT2, B. anthracis Sterne and all clinical isolates essentially as outlined above, with the exception that the assays were conducted in 96-well plates with a final volume of $0.2 \mathrm{ml}$, and concentrations of BAP ranged from 0 to $20 \mathrm{~mm}\left(0-4000 \mu \mathrm{g} \mathrm{ml}^{-1}\right)$. All antimicrobial susceptibility assays were performed in triplicate.

\section{E. coli growth rescue studies}

For the growth rescue experiments, 96-well plates were used with a final culture volume of $0.2 \mathrm{ml}$. The standardized inoculum $(\mathrm{McFarland}=0.5$ ) was prepared in the same manner as stated previously. These plates were then incubated in a SpectramaxPlus 384 plate reader at $37^{\circ} \mathrm{C}$ for $16 \mathrm{~h}$, with shaking at 220 r.p.m. every $30 \mathrm{~min}$ for $15 \mathrm{~min}$. Fractional growth was then determined relative to the no-BAP control, and averaged fractional growth was plotted with standard experimental error as a function of BAP concentration. For the 1-deoxy-D-xylulose (DX) rescue experiments, a range of 0-1.0 mM DX was evaluated in the presence of $0-5.0 \mathrm{~mm}$ BAP in CAMHB media. For the thiamin rescue experiments, a range of $0-1.0 \mathrm{~mm}$ thiamin was evaluated in the presence of 0-0.66 mM BAP in M9 minimal media (no thiamin). DX and thiamin rescue experiments were performed in quadruplicate. DXP synthase overexpression rescue experiments were carried out in LB broth, and the following cell types were treated with 0-5.0 mM BAP: BL21 (no vector), pET37b-BL21, wild-type E. coli $d x s-$ pET37b/BL21 and E370A E. coli $d x s-$ pET37b/BL21. Experiments were performed in triplicate.

DXP synthase overexpression was confirmed by $10 \%$ SDS-PAGE, and incorporation of the octa-histidine tag was verified using the InVision Histag In-gel Stain (Invitrogen, Grand Island, NY, USA) (Supplementary Figure S2). Site-directed mutagenesis of the E370A residue was carried out as reported previously ${ }^{11}$ using the QuikChange site-directed mutagenesis kit (Stratagene, Santa Clara, CA, USA), and the following primers (Integrated DNA Technologies): $5^{\prime}$-CGACGTGGCAATTGCCGCGCAACAGCGGGTGACCTTT GC-3' and 5'-GCAAAGGTCACCGCGTGTTGCGCGGCAATTGCCACGTCG-3'

\section{Checkerboard antibiotic combination studies}

For the checkerboard antibiotic combination studies in E. coli, culture tubes were used with a final culture volume of $0.5 \mathrm{ml}$, prepared as described above. The concentration of ampicillin evaluated for these studies ranged from $0,0.13$ up to $8 \mu \mathrm{g} \mathrm{ml}^{-1}$, tetracycline ranged from $0,0.125$ up to $4 \mu \mathrm{g} \mathrm{ml}^{-1}$ and fosmidomycin ranged from $0,0.19$ up to $6 \mu \mathrm{g} \mathrm{ml}^{-1}$.

For each antibiotic combination, the fractional inhibitory concentration (FIC) index was determined to predict drug synergism: ${ }^{31,32}$

$$
\frac{(B A P)}{\left(M I C_{B A P}\right)}+\frac{(X)}{\left(M I C_{X}\right)}=F C_{B A P}+F I C_{X}=\text { FIC index }(F I C I) .
$$

The FICI was calculated using Equation (1) ${ }^{31,32} \cdot M I C_{B A P}$ and $M I C_{X}$ are the lowest concentrations of $B A P$ or drug $X(X=$ ampicillin, fosmidomycin or tetracycline) showing $\leqslant 15 \%$ growth. The $F I C_{B A P}$ was calculated as the [BAP in the presence of drug $X]$ for a well showing $\leqslant 15 \%$ growth, divided by $M I C_{B A P}$ ${ }_{F I} C_{X}$ was calculated as the $[\operatorname{drug} X$ in the presence of $B A P]$ in the same well, divided by $M I C_{X}$. The FICI is the sum of $F I C_{B A P}$ and $F I C_{X}$. For each combination, the FIC index values were used to indicate drug synergism $(x<0.5)$, additivity $(0.5<x<1.0)$, indifference $(1.0<x<2.0)$ or antagonism $(x>2)$. Fractional growth was determined relative to the no-drug growth control and average values were used. The BAP-ampicillin and BAP-tetracycline checkerboard assays were performed in duplicate. The BAP-fosmidomycin checkerboard assay was performed in quadruplicate.

\section{RESULTS}

BAP selectively inhibits DXP synthase from M. tuberculosis, $Y$. pestis and S. enterica serovar Typhi

Our previous work has established alkyl acetylphosphonates as selective inhibitors of $E$. coli DXP synthase, ${ }^{16,19}$ suggesting that it is possible to achieve selective inhibition of this ThDP-dependent enzyme on the basis of differences in substrate usage and mechanism. ${ }^{11,18,28}$ Here, in vitro inhibition kinetics were performed to characterize BAP inhibitory activity against DXP synthase from M. tuberculosis, S. enterica and Y. pestis (Table 1) to determine the feasibility of this approach across pathogens. Both S. enterica and $Y$. pestis DXP synthase have similar primary amino-acid sequence identity with the E. coli enzyme ( $\sim 90 \%$ ) (Supplementary Table S1). M. tuberculosis DXP synthase (Dxs1) is the least similar to each of the three $(\sim 40 \%)$. All DXP synthase enzymes were kinetically characterized using the enzyme-coupled assays as described previously $^{11}$ and the results are shown in Table 1 . The $K_{\mathrm{m}}$ values obtained for Dxs1 from $M$. tuberculosis in this study $\left(K_{\mathrm{m}}{ }^{\text {pyruvate }}=81 \pm 3 \quad\right.$ and $\left.\quad K_{\mathrm{m}}{ }^{\text {D-GAP }}=16.6 \pm 0.9\right) \quad$ (Supplementary Figure S2) are comparable to those reported previously. ${ }^{8} K_{\mathrm{m}}$ pyruvate and $K_{\mathrm{m}}{ }^{\mathrm{D}-\mathrm{GAP}}$ determined for DXP synthase from $Y$. pestis $\left(K_{\mathrm{m}}{ }^{\text {pyruvate }}=51 \pm 6\right.$ and $\left.K_{\mathrm{m}}{ }^{\mathrm{D}-\mathrm{GAP}}=26 \pm 3\right)$ and $S$. enterica $\left(K_{\mathrm{m}}{ }^{\text {pyruvate }}=76 \pm 19\right.$ and the $\left.K_{\mathrm{m}}{ }^{\mathrm{D}-\mathrm{GAP}}=12 \pm 1\right) \quad$ (Supplementary Figure S2) are also comparable to kinetic constants measured for other DXP synthase enzymes. ${ }^{9,11,33}$

Table 1 Kinetic analysis of DXP synthase from Y. pestis, M. tuberculosis and S. enterica DXP synthase

\begin{tabular}{lllll}
\hline Species & Substrate & $\mathrm{K}_{m}(\mu \mathrm{M})$ & $\mathrm{k}_{\text {cat }}\left(\min ^{-1}\right)$ & $\mathrm{K}_{i}^{B A P}(\mu \mathrm{M})$ \\
\hline Y. pestis & $\begin{array}{l}\text { Pyruvate } \\
\text { D-GAP }\end{array}$ & $51 \pm 6$ & $112 \pm 7$ & $7.5 \pm 0.9$ \\
& $26 \pm 3$ & & \\
M. tuberculosis & Pyruvate & $81 \pm 3$ & $48 \pm 1$ & $4 \pm 2$ \\
& D-GAP & $17 \pm 1$ & & \\
S. enterica & Pyruvate & $76 \pm 19$ & $140 \pm 20$ & $8.4 \pm 0.4$ \\
& D-GAP & $12 \pm 1$ & &
\end{tabular}

Abbreviation: DXP, 1-deoxy-D-xylulose 5-phosphate. $K_{\mathrm{m}}$ is the concentration of substrate giving half maximal velocity in the presence of saturating second substrate. $k_{\mathrm{cat}}$ is the turnover number. $K_{\mathrm{i}}^{\mathrm{BAP}}$ is the dissociation constant for BAP. 
Table 2 Antimicrobial activity of BAP against pathogenic bacteria and FIC index values for antibiotics in combination with BAP

\begin{tabular}{lclc}
\hline Organism & MIC BAP $\left(\mu \mathrm{g} \mathrm{m} \mathrm{l}^{-1}\right)$ & Combination & FIC index \\
\hline Escherichia coli MG1655 & \multirow{2}{*}{1000} & BAP-fosmidomycin & 0.25 \\
& & BAP-ampicillin & 0.31 \\
& & BAP-tetracycline & 0.53 \\
\hline
\end{tabular}

Abbreviations: BAP, butylacetylphosphonate; FIC, fractional inhibitory concentration.

The inhibitory activity of BAP was characterized against each enzyme using the enzyme-coupled assay as described previously ${ }^{19}$ (Supplementary Figure S3), and $K_{\mathrm{i}}$ values are summarized in Table 1. In all cases, low micromolar inhibitory activity of BAP is observed that is comparable to the inhibitory activity of BAP against E. coli DXP synthase. ${ }^{19}$ As expected, the mode of inhibition is competitive with respect to pyruvate and non-competitive with respect to D-GAP (for M. tuberculosis DXP synthase, $K_{\mathrm{i}}^{\mathrm{BAP}}=4 \pm 2 \mu \mathrm{M}$; for Y. pestis DXP synthase, $K_{\mathrm{i}}^{\mathrm{BAP}}=7.5 \pm 0.9 \mu \mathrm{M} ;$ for $S$. enterica DXP synthase, $\left.K_{\mathrm{i}}^{\mathrm{BAP}}=8.4 \pm 0.4 \mu \mathrm{M}\right)$.

\section{BAP exhibits weak antimicrobial activity}

As a starting point, BAP was evaluated for antimicrobial activity against the E. coli ATCC strain MG 1655 using the macrodilution method. $^{31,32}$ BAP exhibits MICs $(M I C=[B A P]$ causing growth inhibition) of $\sim 1000 \mu \mathrm{g} \mathrm{ml}^{-1}$ in CAMHB and a significantly lower MIC of $122 \mu \mathrm{g} \mathrm{ml}^{-1}$ in M9 minimal media (Table 2). BAP was evaluated against several clinically isolated pathogens (Supplementary Table S2), and BAP exhibits an MIC of $4000 \mu \mathrm{g} \mathrm{ml}^{-1}$ against S. enterica serovar Typhimurium and Micrococcus sp., $1000 \mu \mathrm{g} \mathrm{ml}^{-1}$ against B. anthracis Sterne and $4000 \mu \mathrm{g} \mathrm{ml}^{-1}$ against Pseudomonas aeruginosa in CAMHB.

We considered the possibility that the weak antimicrobial activity of BAP is attributed to poor cellular uptake. Small phosphorylated (or phosphonylated) molecules, including the phosphonate fosmidomycin, are actively transported via the glycerol 3-phosphate transporter $(g l p T) .^{34-37}$ BAP was evaluated for antimicrobial activity against a glpT knockout strain (JW2234-2) and its parent strain (BW25513) (Yale, Coli Genetic Stock Center) to determine whether this is a possible uptake mechanism. However, while fosmidomcyin is inactive against the $g l p T$ knockout strain, BAP exhibits comparable antimicrobial activity against the $g l p T$ knockout and parent strains (Supplementary Figure S4). This result indicates the glycerol 3-phosphate transporter is not likely a mechanism of entry for BAP.

DXP synthase is an intracellular target of BAP

Experiments to rescue the growth inhibitory effects of BAP against E. coli MG1655 were performed to provide evidence for DXP synthase as an intracellular target.

Rescue by downstream metabolites

Previous reports demonstrate labeling of downstream MEP pathway intermediates in E. coli in feeding experiments using labeled DX, suggesting DX is cell permeable and undergoes intracellular phosphorylation at some level to produce DXP. ${ }^{38,39}$ Here, partial rescue of BAP inhibitory activity is observed when the growth medium is supplemented with DX (50\% reduction in growth inhibition by BAP in the presence of DX) (Figure 3a). As DXP synthase is a branch point in bacterial metabolism, additional biosynthetic pathways including ThDP and pyridoxal phosphate biosynthesis ${ }^{1,4,40}$ should be affected
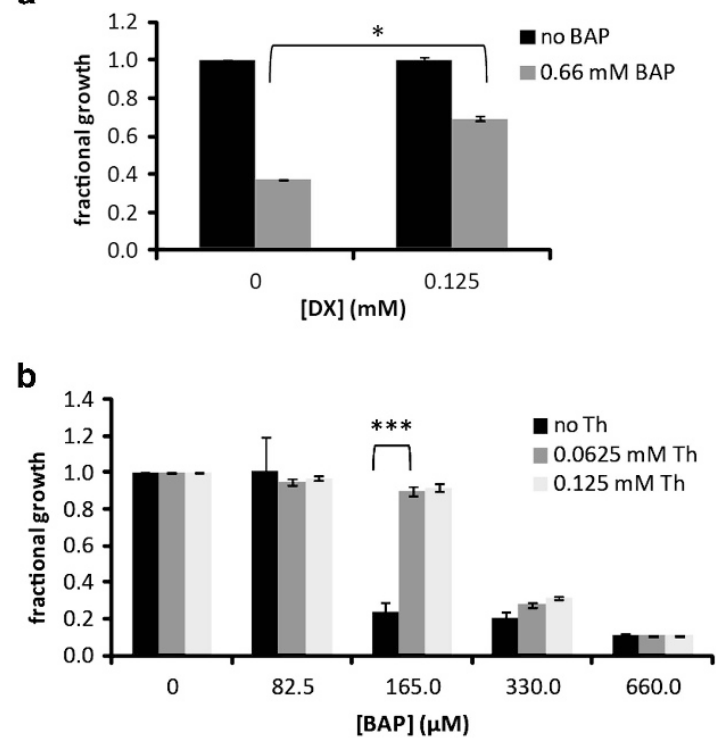

C
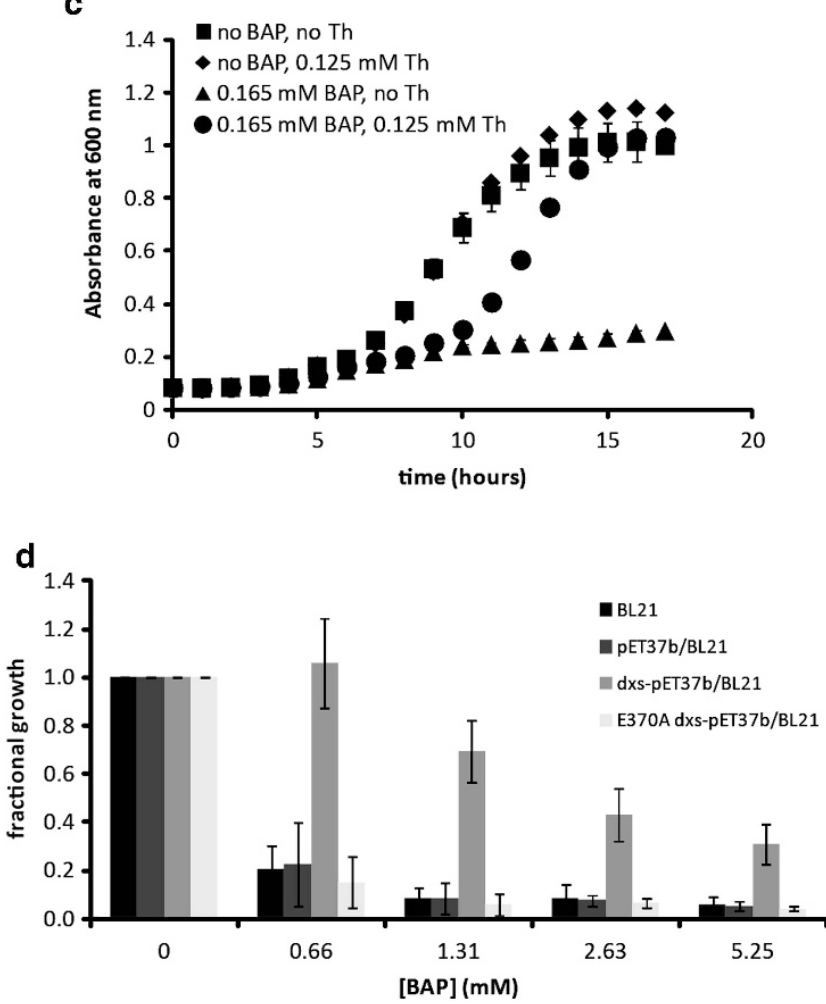

Figure 3 Butylacetylphosphonate (BAP) inhibition of E. coli MG1655 growth is rescued by 1-deoxy-D-xylulose (DX), thiamin (Th) and E. coli DXP synthase (Dxs) overexpression. (a) Fractional growth at $12.5 \mathrm{~h}$ in the presence and absence of BAP and DX. Mean and standard error of four replicates are shown. ${ }^{*} P=0.022$ (unpaired $t$-test comparing DX-treated and -untreated cultures at $0.66 \mathrm{~mm}$ BAP). (b) Fractional growth at $16 \mathrm{~h}$ in the presence and absence of BAP and thiamin. Mean and standard error of four replicates is shown. ${ }^{* * *} P=0.0000258$ (unpaired $t$-test comparing thiamintreated and -untreated cultures at $165 \mu \mathrm{m} \mathrm{BAP).} \mathrm{(c)} \mathrm{Growth} \mathrm{curve} \mathrm{over} 16 \mathrm{~h}$, in the absence or presence of BAP and thiamin. (d) Fractional growth at $16 \mathrm{~h}$ determined for $E$. coli BL21 cells only, cells transformed with empty $\mathrm{pET} 37 \mathrm{~b}$ vector, vector-expressing recombinant $E$. coli DXP synthase protein (dxs-pET37b) or vector expressing the catalytically inactive ${ }^{15}$ E370A variant (E370A dxs-pET37b). Mean and standard error of four replicates is shown. 
by inhibition of DXP synthase. Further, inhibition of ThDP biosynthesis might potentiate the loss of DXP synthase activity as this cofactor is also required for DXP synthase catalysis. Here, complete rescue of growth inhibitory effects of $31.25 \mu \mathrm{g} \mathrm{ml}^{-1}$ BAP $(165 \mu \mathrm{M})$ is observed in the presence of thiamin (Figure $3 \mathrm{~b}$ ). E. coli growth rates in the presence or absence of thiamin are similar (Figure 3c), suggesting that intracellular thiamin concentration is sufficient to support normal growth under conditions where ThDP biosynthesis is not inhibited. Although growth of BAP-treated cells is restored by $16 \mathrm{~h}$ in the presence of thiamin, there is a clear delay in growth under these conditions. This may reflect the time required for intracellular phosphorylation of thiamin under growth inhibitory conditions. Under conditions of higher BAP concentration, thiamin supplementation does not rescue growth by $16 \mathrm{~h}$ (Figure 3c).

\section{Rescue by DXP synthase overexpression}

Overexpression of DXP synthase should rescue growth of BAP-treated E. coli ${ }^{41}$ if BAP acts by inhibition of DXP synthase. Indeed, increasing intracellular levels of DXP synthase (Supplementary Figure S1) results in rescue of bacterial growth (Figure 3d) compared with E. coli harboring empty vector or the catalytically inactive E370A mutant. ${ }^{15}$

\section{Growth inhibition is significantly enhanced when BAP is administered in antibiotic combinations}

The weak antimicrobial activity of BAP on its own prompted a study to determine the synergistic effects of BAP in combination with several established antibiotics. As a starting point, we have investigated synergistic effects of BAP in combination with fosmidomycin, ampicillin and tetracycline using the checkerboard method. ${ }^{31,32}$ Fosmidomycin potently inhibits the second enzyme in the MEP pathway, MEP synthase (IspC) (Figure 1). ${ }^{42-44}$ Given the established synergy that can take place between agents that target the same metabolic pathway, and in particular the synergy observed between fosmidomycin and inhibitors of isoprenoid biosynthesis enzyme farnesylpyrophosphate synthase, ${ }^{45}$ we hypothesized that BAP could synergize with fosmidomycin. Likewise, fosmidomycin has been shown to synergize with cell wall biosynthesis inhibitors, including ampicillin, to inhibit bacterial cell growth. ${ }^{46}$ Those early observations established precedence for drug synergisms between agents targeting isoprenoid biosynthesis and cell wall biosynthesis (which relies upon the prenylated lipid II) and suggest that synergy between BAP and cell wall biosynthesis inhibitors is also possible. Finally, we considered the possibility that synergy could exist between BAP and the bacterial translation inhibitor tetracycline, on the basis that geranylation of tRNA is proposed to have a role to modulate codon recognition during translation, ${ }^{47}$ thus drawing a connection between isoprenoid biosynthesis and protein synthesis.

Here, the FIC index was determined for BAP-fosmidomycin, BAPampicillin and BAP-tetracycline combinations against E. coli MG1655 to predict drug synergisms. ${ }^{31,32}$ On the basis of this analysis, the antimicrobial activity of BAP appears to be enhanced in combination with fosmidomycin $(F I C I=0.25$; Table 2$)$ or ampicillin $(F I C I=0.31$; Table 2). Analysis by isobolograms suggests pronounced synergy in the BAP-fosmidomycin combination against E. coli MG1655 (Supplementary Figure S5). ${ }^{31,48}$ However, examination of the BAPampicillin combination by isobologram indicates synergy is less pronounced, suggesting this combination may display additive effects. The FICI for BAP in combination with tetracycline is 0.53 , indicating an additive relationship (Table 2).

\section{DISCUSSION}

As the isoprenoid biosynthetic pathway is absent in mammals, but essential in many human pathogens, each of the seven enzymes in the pathway represents a prospective antibiotic target. Selective inhibition of DXP synthase could reduce flux through multiple metabolic pathways, and this represents a potential advantage of targeting the first step of the MEP pathway. Here we have evaluated the inhibition and antimicrobial activity of BAP, a DXP synthase inhibitor developed in our lab to target selectively the uniquely large active site of this enzyme. ${ }^{19}$

BAP displays low micromolar inhibitory activity against DXP synthase from M. tuberculosis, S. enterica and Y. pestis, and this is consistent with the idea that BAP targets a mechanism common to all pathogenic DXP synthase enzymes, but distinct from mammalian ThDP-dependent enzymes. Not surprisingly, BAP acts as a competitive inhibitor with respect to pyruvate in all cases, indicating that the acetylphosphonate moiety of BAP acts as a pyruvate mimic in this unnatural bisubstrate analog.

BAP appears to exert antimicrobial activity against E. coli MG1655 through a mechanism that involves DXP synthase inhibition, as suggested by the observed rescue of its inhibitory effects in the presence of DX or thiamin, or under conditions of DXP synthase overexpression. Partial rescue is observed using non-phosphorylated DX, implying DXP synthase as a target. More pronounced rescue is observed in the presence of thiamin or DXP synthase overexpression. In addition to rescuing the thiamin pathway and other thiamindependent cellular processes under these conditions, it is possible that ThDP generated under thiamin rescue conditions regenerates active DXP synthase by competing with butylphosphonolactyl-thiamin disphosphate (butyl phosphonolactyl-ThDP) at the ThDP binding site. Alternatively, increasing intracellular ThDP levels could activate an apo-DXP synthase pool that presumably arises under conditions of DXP synthase inhibition and subsequent cofactor depletion. Not surprisingly, overexpression of active DXP synthase in E. coli also results in pronounced rescue of BAP growth inhibitory activity. The observation that rescue is less pronounced under all of these conditions with increasing BAP is consistent with DXP synthase inhibition coupled with other nonspecific antimicrobial effects of BAP at high concentrations.

The reason for the observed weak antimicrobial activity of BAP on its own against the pathogens tested here is not entirely clear, although poor cell permeability is conceivable. Recent studies by Cirello et al. ${ }^{49}$ reveal the natural product dehydrophos as a prodrug of the structurally related methyl acetylphosphonates, a mimic of pyruvate and known inhibitor of the ThDP-dependent enzymes that catalyze decarboxylation of pyruvate. ${ }^{10,20-22}$ This phosphonotripeptide natural product is imported via oligopeptide permeases and cleaved by intracellular peptidases to release methyl acetylphosphonates. By extension, the acetylphosphonate scaffold of BAP may hamper efficient uptake in the absence of such a delivery mechanism. It is intriguing to contemplate a similar delivery strategy for enhancing cellular uptake of BAP and analogs, although it is also possible that incorporation of more hydrophobic substituents may enhance permeability.

Despite the weak antimicrobial effects of BAP on its own, BAPfosmidomycin and BAP-ampicillin combinations are synergistic against E. coli MG1655. The synergistic BAP-fosmidomycin combination was anticipated, given that drug synergism in the MEP pathway has been previously reported with farnesylpyrophosphate synthase inhibitors and fosmidomycin. ${ }^{45}$ Our previous work suggesting MEP activates and sustains IspF activity in a possible feedforward 
regulatory mechanism in bacterial isoprenoid biosynthesis ${ }^{50}$ could provide additional basis for this synergistic combination. It is conceivable that inhibition of upstream enzymes DXP synthase and IspC by BAP and fosmidomycin, respectively, not only reduces flux through the pathway at these points but also effectively expedites the loss of IspF activity by rapidly depleting MEP levels. There is established precedence for drug synergisms between inhibitors of isoprenoid biosynthesis and cell wall biosynthesis, ${ }^{45,51}$ given the requirement for isoprenoid precursors in lipid II biosynthesis, ${ }^{41}$ and so the enhanced activity observed with the BAP-ampicillin combination in this study was also anticipated. It is also plausible that ampicillin compromises cell wall integrity to permit more efficient uptake of BAP. These findings are significant given the necessity of combination therapies to prevent emergence of antimicrobial resistance. ${ }^{52}$ Although inhibition of DXP synthase ought to simultaneously reduce flux through multiple essential pathways, the emergence of mechanisms to circumvent such an intervention in bacteria is inevitable. ${ }^{52-54}$

This work highlights unnatural bisubstrate analogs as selective inhibitors of this unique ThDP-dependent enzyme and promising antimicrobial agents as components of synergistic antimicrobial combinations. Although the acetylphosphonates were developed over 30 years ago as ketoacid mimics, and are powerful tools to study ThDP-dependent enzymes, ${ }^{10,20}$ in the case of dehydrophos, it is clear that Nature has already conceived of such an antimicrobial strategy to inhibit essential ThDP-dependent processes. Development of acetylphosphonates to increase selectivity, potency and permeability is perhaps a new chapter in medicinal chemistry on natural product scaffolds.

\section{ACKNOWLEDGEMENTS}

We gratefully acknowledge Leighanne Basta for providing the E370A DXP synthase variant and Paul Phillips for assistance in evaluating the antimicrobial activity of BAP. Eric Nuermberger is acknowledged for helpful insight and for providing M. tuberculosis genomic DNA. We thank Theresa Shapiro and Jürgen Bosch for helpful discussions. This work was supported by The National Institute of Health: 5T32-GM007445 (JMS) and T32GM08018901 (RJV), R01GM084998 (CLFM, RJV, JMS) and F31GM099467 (JMS). This project has been funded in part with Federal funds from the National Institute of Allergy and Infectious Diseases, National Institutes of Health, Department of Health and Human Services, under Contract Nos. HHSN272200700058C and HHSN272201200026C (MLK, WFA).

1 Arigoni, D. et al. Terpenoid biosynthesis from 1-deoxy-D-xylulose in higher plants by intramolecular skeletal rearrangement. Proc. Natl Acad. Sci. USA 94, 10600-10605 (1997).

2 Rohmer, M., Knani, M., Simonin, P., Sutter, B. \& Sahm, H. Isoprenoid biosynthesis in bacteria: a novel pathway for the early steps leading to isopentenyl diphosphate. Biochem. J. 15, 517-524 (1993).

3 Walsh, C. T. Antibiotics: Actions, Origins, Resistance (ASM Press, Washington, DC, USA, 2003).

4 Sprenger, G. A. et al. Identification of a thiamin-dependent synthase in Escherichia coli required for the formation of the 1-deoxy-D-xylulose 5-phosphate precursor to isoprenoids, thiamin, and pyridoxol. Proc. Natl Acad. Sci. USA 94, 12857-12862 (1997).

5 Lois, L. M. et al. Cloning and characterization of a gene from Escherichia coli encoding a transketolase-like enzyme that catalyzes the synthesis of D-1-deoxyxylulose 5phosphate, a common precursor for isoprenoid, thiamin, and pyridoxol biosynthesis. Proc. Natl Acad. Sci. USA 95, 2105-2110 (1998).

6 Kuzuyama, T., Takagi, M., Takahashi, S. \& Seto, H. Cloning and characterization of 1 deoxy-D-xylulose 5-phosphate synthase from Streptomyces sp. strain CL190, which uses both the mevalonate and nonmevalonate pathways for isopentenyl diphosphate biosynthesis. J. Bacteriol. 15, 891-897 (2000).

7 Hahn, F. M. et al. 1-Deoxy-D-xylulose 5-phosphate synthase, the gene product of open reading frame (ORF) 2816 and ORF 2895 in Rhodobacter capsulatus. J. Bacteriol. 183, 1-11 (2001).
8 Bailey, A. M., Mahapatra, S., Brennan, P. J. \& Crick, D. C. Identification, cloning purification, and enzymatic characterization of Mycobacterium tuberculosis 1-deoxy-Dxylulose 5-phosphate synthase. Glycobiology 12, 813-820 (2002).

9 Lee, J., Oh, D. \& Kim, S. Cloning and characterization of the $d x s$ gene, encoding 1-deoxy-D-xylulose 5-phosphate synthase from Agrobacterium tumefaciens, and its overexpression in Agrobacterium tumefaciens. J. Biotechnol. 128, 555-566 (2007).

10 O'Brien, T. A., Kluger, R., Pike, D. C. \& Gennis, R. B. Phosphonate analogues of pyruvate. Probes of substrate binding to pyruvate oxidase and other thiamin pyrophosphate-dependent decarboxylases. Biochim. Biophys. Acta 613, 10-17 (1980).

11 Brammer, L. A., Smith, J. M. \& Meyers, C. F. 1-Deoxy-D-xylulose 5-phosphate synthase catalyzes a novel random sequential mechanism. J. Biol. Chem. 42, 36522-36531 (2011).

12 Altincicek, B. et al. Tools for discovery of inhibitors of the 1-deoxy-D-xylulose 5-phosphate (DXP) synthase and DXP reductoisomerase: an approach with enzymes from the pathogenic bacterium Pseudomonas aeruginosa. FEMS Microbiol. Lett. 190, 329-333 (2000).

$13 \mathrm{Mao}$, J. et al. Structure-activity relationships of compounds targeting Mycobacterium tuberculosis 1-deoxy-D-xylulose 5-phosphate synthase. Bioorg. Med. Chem. Lett. 18, 5320-5323 (2008).

14 Matsue, Y. et al. The herbicide ketoclomazone inhibits 1-deoxy-D-xylulose 5-phosphate synthase in the 2-C-methyl-D-erythritol 4-phosphate pathway and shows antibacterial activity against Haemophilus influenzae. J. Antibiot. 63, 583-588 (2010).

15 Xiang, S., Usunow, G., Lange, G., Busch, M. \& Tong, L. Crystal structure of 1-deoxy-Dxylulose 5-phosphate synthase, a crucial enzyme for isoprenoids biosynthesis. J. Biol. Chem. 282, 2676-2682 (2007).

16 Morris, F., Vierling, R., Boucher, L., Bosch, J. \& Freel Meyers, C. L. DXP synthasecatalyzed $\mathrm{C}-\mathrm{N}$ bond formation: nitroso substrate specificity studies guide selective inhibitor design. ChemBioChem 14, 1309-1315 (2013).

17 Eubanks, L. M. \& Poulter, C. D. Rhodobacter capsulatus 1-deoxy-D-xylulose 5phosphate synthase: steady-state kinetics and substrate binding. Biochemistry 42, 1140-1149 (2003).

18 Patel, H., Nemeria, N. S., Brammer, L. A., Meyers, C. F. \& Jordan, F. Observation of thiamin-bound intermediates and microscopic rate constants for their interconversion on 1-deoxy-D-xylulose 5-phosphate synthase: 600-fold rate acceleration of pyruvate decarboxylation by D-glyceraldehyde-3-phosphate. J. Am. Chem. Soc. 44, 18374-18379 (2013).

19 Smith, J. M., Vierling, R. J. \& Meyers, C. F. Selective inhibition of E. coli 1-deoxy-Dxylulose-5-phosphate synthase by acetylphosphonates. Med. Chem. Commun. 3, 65-67 (2012).

20 Kluger, R. \& Pike, D. C. Active site generated analogs of reactive intermediates in enzymic reactions. Potent inhibition of pyruvate dehydrogenase by a phosphonate analog of pyruvate. J. Am. Chem. Soc. 13, 4504-4506 (1977).

21 Gish, G., Smyth, T. \& Kluger, R. Thiamin diphosphate catalysis. Mechanistic divergence as a probe of substrate activation of pyruvate decarboxylase. J. Am. Chem. Soc. 18, 6230-6234 (1988).

22 Bearne, S. L. \& Kluger, R. Phosphoenol acetylphosphonates: substrate analogues as inhibitors of phosphoenolpyruvate enzymes. Bioorg. Chem. 20, 135-147 (1992).

23 Arjunan, P. et al. A thiamin-bound, pre-decarboxylation reaction intermediate analogue in the pyruvate dehydrogenase E1 subunit induces large scale disorder-to-order transformations in the enzyme and reveals novel structural features in the covalently bound adduct. J. Biol. Chem. 281, 15296-15303 (2006).

24 Nemeria, N. S., Korotchkina, L. G., Chakraborty, S., Patel, M. S. \& Jordan, F. Acetylphosphinate is the most potent mechanism-based substrate-like inhibitor of both the human and Escherichia coli pyruvate dehydrogenase components of the pyruvate dehydrogenase complex. Bioorg. Chem. 34, 362-379 (2006).

25 Kale, S., Arjunan, P., Furey, W. \& Jordan, F. A dynamic loop at the active center of the Escherichia coli pyruvate dehydrogenase complex E1 component modulates substrate utilization and chemical communication with the E2 component. J. Biol. Chem. 282, 28106-28116 (2007)

26 Flournoy, D. S. \& Frey, P. A. Inactivation of the pyruvate dehydrogenase complex of Escherichia coli by fluoropyruvate. Biochemistry 25, 9594-9602 (1989).

27 Nemeria, N. et al. The $1^{\prime}, 4^{\prime}$-iminopyrimidine tautomer of thiamin diphosphate is poised for catalysis in asymmetric active centers on enzymes. Proc. Natl Acad. Sci. USA 104, 78-82 (2007).

28 Brammer, L. A. \& Meyers, C. F. Revealing substrate promiscuity of 1-deoxy-D-xylulose 5-phosphate synthase. Org. Lett. 20, 4748-4751 (2009).

29 Dieckman, L., Gu, M., Stols, L., Donnelly, M. I. \& Collart, F. R. High throughput methods for gene cloning and expression. Protein Expr. Purif. 25, 1-7 (2002).

30 Stols, L. et al. A new vector for high-throughput, ligation-independent cloning encoding a tobacco etch virus protease cleavage site. Protein Expr. Purif. 25, 8-15 (2002).

31 Moody, J. in Clinical Microbiology Procedures Handbook Vol. 2 (ed. Isenberg, H. D.) 5.12.1-5.12.23 (American Society for Microbiology, Washington, DC, USA, 2004).

32 Pillai, S. K., Moellering, R. C. \& Eliopoulos, G.M. Antimicrobial Combinations. Antibiotics in Laboratory Medicine (ed Lorian, V.) (Lippincott Williams \& Wilkins, 2005).

33 Kuzuyama, T., Takagi, M., Takahashi, S. \& Seto, H. Cloning and characterization of 1-deoxy-D-xylulose 5-phosphate synthase from Streptomyces sp. Strain CL190, which uses both the mevalonate and nonmevalonate pathways for isopentenyl diphosphate biosynthesis. J. Bacteriol. 182, 891-897 (2000).

34 Venkateswaran, P. S. \& Wu, H. C. Isolation and characterization of a phosphonomycinresistant mutant of Escherichia coli K-12. J. Bacteriol. 110, 935-944 (1972).

35 McKenney, E. S. et al. Lipophilic prodrugs of FR900098 are antimicrobial against Francisella novicida in vivo and in vitro and show GIpT independent efficacy. PLoS One 10, 38167 (2012). 
36 Brown, A. C., Eberl, M., Crick, D. C., Jomaa, H. \& Parish, T. The nonmevalonate pathway of isoprenoid biosynthesis in Mycobacterium tuberculosis is essential and transcriptionally regulated by Dxs. J. Bacteriol. 192, 2424-2433 (2010).

37 Sakamoto, Y., Furukawa, S., Ogihara, H. \& Yamasaki, M. Fosmidomycin resistance in adenylate cyclase deficient (cya) mutants of Escherichia coli. Biosci. Biotechnol. Biochem. 67, 2030-2033 (2003).

38 Giner, J., Giner, J., Jaun, B. \& Arigoni, D. Biosynthesis of isoprenoids in Escherichia coli: the fate of the $3-\mathrm{H}$ and $4-\mathrm{H}$ atoms of 1-deoxy-D-xylulose. Chem. Commun. 1857-1858 (1998).

39 Wungsintaweekul, J. et al. Phosphorylation of 1-deoxy-D-xylulose by D-xylulokinase of Escherichia coli. Eur. J. Biochem. 268, 310-316 (2001).

$40 \mathrm{Hill}, \mathrm{R}$. E. et al. The biogenetic anatomy of vitamin B6. A ${ }^{13} \mathrm{C}$ NMR investigation of the biosynthesis of pyridoxolin in E. coli. J. Biol. Chem. 271, 30426-30435 (1996).

41 Campbell, T. L. \& Brown, E. D. Characterization of the depletion of 2-C-methyl-Derythritol-2,4-cyclodiphosphate synthase in Escherichia coli and Bacillus subtilis. J. Bacteriol. 184, 5609-5618 (2002)

42 Okuhara, M. et al. Studies on new phosphonic acid antibiotics. J. Antibiot. 33, 13-17 (1980)

43 Kuroda, Y. et al. Studies on new phosphonic acid antibiotics: 4. Structure determination of FR-33289, FR-31564 and FR-32863. J. Antibiot. 33, 29-35 (1980).

44 Kuzuyama, T., Shimizu, T., Takahashi, S. \& Seto, H. Fosmidomycin, a specific inhibitor of 1-deoxy-D-xylulose 5-phosphate reductoisomerase in the nonmevalonate pathway for terpenoid biosynthesis. Tetrahedron Lett. 39, 7913-7916 (1998).
45 Leon, A. et al. Isoprenoid biosynthesis as a drug target: bisphosphonate inhibition of Escherichia coli K12 growth and synergistic effects of fosmidomycin. J. Med. Chem. 25, 7331-7341 (2006).

$46 \mathrm{Neu}, \mathrm{H}$. \& Kamimura, T. Synergy of fosmidomycin (FR-31564) and otherantimicrobial agents. Antimicrob. Agents Chemother. 22, 560-563 (1982).

47 Dumelin, C., Chen, Y., Leconte, A., Chen, Y. \& Liu, D. Discovery and biological characterization of geranylated RNA in bacteria. Nat. Chem. Biol. 11, 913-919 (2012).

48 Kalan, L. \& Wright, G. D. Antibiotic adjuvants: multicomponent anti-infective strategies. Expert Rev. Mol. Med. 13, e5 (2011).

49 Circello, B., Miller, C., Lee, J., van der Donk, W. \& Metcalf, W. The antibioticdehydrophos is converted to a toxic pyruvate analog by peptide bond cleavage in Salmonella enterica. Antimicrob. Agents Chemother. 55, 3357-3362 (2011).

50 Bitok, J. \& Meyers, C. F. 2C-methyl-D-erythritol 4-phosphate enhances and sustains cyclodiphosphate synthase IspF activity. ACS Chem. Biol. 7, 1702-1710 (2012).

51 Zhu, W. et al. Antibacterial drug leads targeting isoprenoid biosynthesis. Proc. Natl Acad. Sci. USA 110, 123-128 (2013).

52 Walsh, C. T. \& Wencewicz, T. A. Prospects for new antibiotics: a molecule-centered perspective. J. Antibiot. doi:10.1038/ja.2013.49 (in press).

$53 \mathrm{Erb}, \mathrm{T}$. et al. A RubisCO-like protein links SAM metabolism with isoprenoid biosynthesis. Nat. Chem. Biol. 8, 926-932 (2012).

54 Perez-Gil, J. et al. Mutations in Escherichia coli aceE and ribB genes allow survival of strains defective in the first step of the isoprenoid biosynthesis pathway. PLoS One 8, e43775 (2012)

Supplementary Information accompanies the paper on The Journal of Antibiotics website (http://www.nature.com/ja) 